\title{
Toponimi e identità: la percezione dei parlanti valdostani (Prima Parte)
}

\section{Luisa Revelli}

\section{(2) OpenEdition}

\section{Journals}

Edizione digitale

URL: http://journals.openedition.org/esp/2727

DOI: $10.4000 /$ esp. 2727

ISSN: 2532-0319

\section{Editore}

Centre d'Information sur l'Éducation Bilingue et Plurilingue

\section{Edizione cartacea}

Data di pubblicazione: 1 décembre 2013

Paginazione: 15-27

ISSN: 1127-266X

\section{Notizia bibliografica digitale}

Luisa Revelli, «Toponimi e identità: la percezione dei parlanti valdostani (Prima Parte)», Éducation et sociétés plurilingues [Online], 35 | 2013, Messo online il 01 février 2020, consultato il 17 février 2021. URL: http://journals.openedition.org/esp/2727 ; DOI: https://doi.org/10.4000/esp.2727 


\section{TOPONIMI E IDENTITÀ: LA PERCEZIONE DEI PARLANTI VALDOSTANI (PRIMA PARTE)}

\section{Luisa REVELLI}

Les noms de lieux de la Vallée d'Aoste reproduisent les phénomènes historiques de la stratification plurilingue: bien qu'ayant fait l'objet d'interventions de normalisation graphique, encore aujourd'hui - et même à certains égards, aujourd'hui plus que jamais - ils sont exposés dans l'oralité à une prolifération de variantes représentatives des profils linguistiques diversifiés des locuteurs. L'article présente les résultats d'une enquête menée en VDA dans le but de recenser les differentes réalisations phonétiques d'une sélection de noms de lieux, de corréler les formes concurrentes aux caractéristiques personnelles et sociolinguistiques des locuteurs, de détecter les perceptions de connexion entre types de prononciation et évaluations d'exactitude, crédibilité sociale, stigmatisation, prestige et enfin de remarquer la possible valeur symbolique et identitaire attribuée aux toponymes comme réferents d'une présumée fidélité aux traditions linguistiques et culturelles de la région.

Placenames in the Aosta Valley reflect the historical phenomena of plurilingual stratification: though they underwent a process of written normalization, today - and sometimes more than ever - they are subject, in spoken language, to a proliferation of variations representing the varied linguistic profiles of speakers. The article presents the results of a survey carried out in the Valley in order to count all the different phonetic realizations of a selection of placenames, to correlate competing forms with speakers' personal and sociolinguistic characteristics, to detect the connections between types of pronunciation and evaluations of exactitude, social credibility, stigmatization, prestige and lastly to note the possible symbolic and identity markers attributed to topomyms as a reference to a supposed loyalty to the linguistic and cultural traditions of the region.

PREMESSE: LA TOPONOMASTICA IN VALLE D'AOSTA differenza di quanto avvenuto in molte altre regione italiane, in A cui la presenza di parlate minoritarie o seconde lingue ufficiali 1 ha dato origine a soluzioni ufficiali alternative per le diverse trafile, in Valle d'Aosta i toponimi dispongono formalmente di un'unica forma che costituisce l'esito di una tradizione nel corso dei secoli esposta a rese oscillanti o arbitrarie indotte dalla complessità dei processi interlinguistici di conversione dalla sfera orale, dominata dal francoprovenzale, a quella scritta, veicolata dal latino prima, dal francese poi, dall'italiano infine (Raimondi 2003, 2006).

Con l'obiettivo di individuare soluzioni grafiche uniformi e al contempo volte al recupero delle forme ritenute più fedeli alla tradizione, con un recente provvedimento normativo (1) il governo regionale ha nominato e affidato a una Commissione il compito di elaborare i "Critères à suivre pour la graphie des dénominations officielles", criteri che hanno sancito un principio di base secondo cui le grafie dei toponimi, 
Toponimi e identità: la percezione dei parlanti valdostani (Prima Parte)

L. ReVelui basate sulle convenzioni ortografiche del francese, devono tenere conto della tradizione orale di matrice francoprovenzale e prevedere accorgimenti quali la conservazione o restituzione $\mathrm{di}-\mathrm{z}$, $-\mathrm{x} \mathrm{e}-\mathrm{d}$ finali diacritiche (2).

Al tentativo di ristandardizzazione delle denominazioni ufficiali si sono intanto affiancati interventi sulla microtoponomastica messi in atto dalle singole amministrazioni municipali: il Comune di Aosta ha, per esempio, recentemente provveduto a modificare la denominazione di alcune zone urbane e a ripristinare nelle targhe delle vie del centro le grafie francesi settecentesche. Alcune di queste iniziative - come la francesizzazione della denominazione di quartieri nati e storicamente indicati con appellativi italiani (ad es. Quartiere Dora, diventato Quartierde-la-Doire) o la traduzione nella lingua d'Oltralpe dei primi nomi dei personaggi cui le vie sono intitolate (ad es. Antonio Gramsci, diventato Antoine Gramsci) - hanno acceso vivaci dibattiti politici che però non sembrano aver coinvolto né tanto meno appassionato la comunità dei parlanti. La pronuncia dei toponimi da parte di questi ultimi è del resto caratterizzata da un bassissimo livello di codificazione: le scelte che contraddistinguono i comportamenti orali appaiono svincolate dalle soluzioni scrittorie tramandate e/o istituzionalmente validate e lontane da un'idea di norma riconducibile ad un sistema linguistico univoco. Negli enunciati dei parlanti valdostani un medesimo referente toponimico può, infatti, dare origine a svariate soluzioni denominative antagoniste. Non si tratta però mai di trasposizioni in direzione della traduzione da un codice all'altro: semplicemente, le forme di base, mantenendo i tratti caratterizzanti della matrice francese, vengono reinterpretate in modo diversificato a seconda che il modello fonologico e le norme di conversione dallo scritto al parlato si orientino prevalentemente sull'italiano, lingua oggi prevalente in tutti domini (Berruto 2003), al francoprovenzale, codice endogeno in consistente fase di rivalutazione anche nella prospettiva del particolarismo autonomista, o al francese, statutariamente seconda lingua ufficiale della regione, veicolata dall'insegnamento scolare e all'interno della comunità rappresentativa di valori simbolici di alterità e prestigio (Puolato 2006).

Le diverse varianti concorrenti si alternano così, tanto nei contesti informali quanto in quelli pubblici (trasmissioni radio-televisive locali, discorsi politici, ecc.), più o meno pacificamente convivendo a livello individuale e comunitario.

Nasce su queste basi l'idea di censire, attraverso un'indagine rappresentativa di molteplici categorie di parlanti, le polimorfe rese orali per uno stesso toponimo, e di elicitare, per mezzo di interviste mirate, i livelli di consapevolezza e le idee di norma sottese alle singole scelte di pronuncia, le potenziali rappresentazioni di prestigio, stigma, inadeguatezza e 
Toponimi e identità: la percezione dei parlanti valdostani (Prima Parte)

L. ReVELLI

\section{L'INCHIESTA:} ASPETTI METODOLOGICI le esplicite e implicite valenze identitarie attribuite a specifiche soluzioni: se riconosciamo, infatti, la natura funzionale della differenziazione delle pronunce regionali italiane, accogliendo l'ipotesi che la loro resilienza alla standardizzazione "serva a strutturare i gruppi e contribuisca attivamente a costituire l'identità degli individui" (Antonini-Moretti 2000: 12), la correlazione tra i comportamenti linguistici e i modelli normativi rispettivamente adottati e dichiarati dai parlanti valdostani per la pronuncia delle denominazioni di luogo costituisce un oggetto di studio particolarmente interessante, potendo in un contesto di italofonia così profondamente mosso dalla stratificazione plurilingue i toponimi assumere il ruolo di referenti simbolici di un'identità asserita per esibire lealtà linguistica e culturale alla tradizione del territorio, e quindi per affermare - in presenza o a maggior ragione in assenza di abitudini linguistiche individuali al particolarismo conformi - sentimenti di inclusione e appartenenza alla comunità.

L'inchiesta, condotta sul campo da Hélène Champvillair nel periodo compreso tra l'ottobre 2012 e il marzo 2013, ha coinvolto 135 parlanti, omogeneamente suddivisi per genere, ripartiti in tre macroclassi di età (3) e provenienti da diverse aree della regione (4). Il campione è risultato sufficientemente variegato in relazione al titolo di studio posseduto (5) e rappresentativo delle abitudini linguistiche tipiche della Valle d'Aosta contemporanea: il $70 \%$ degli intervistati ha, infatti, dichiarato di utilizzare nel contesto familiare l'italiano, mentre il $30 \%$ ha affermato di usare il francoprovenzale, da solo $(9 \%)$, in alternanza con l'italiano o in combinazione con altri codici.

L'intervista è stata condotta interamente in italiano, e articolata in due parti, attraverso la combinazione di tecniche escussive complementari. Nella prima sezione a ciascun parlante è stata presentata una mappa della Valle d'Aosta ripartita secondo i confini rappresentativi dei territori comunali. Alla mappa mancavano le etichette relative a 16 dei 74 Comuni valdostani: gli intervistati venivano quindi invitati a denominare i toponimi mancanti, possibilmente menzionando anche quelli confinanti. La natura topografica del compito, che induceva gli intervistati a concentrare tutta l'attenzione sulla collocazione geografica delle aree comunali, ha consentito di ottenere realizzazioni poco o affatto foneticamente sorvegliate.

Una volta illustrato lo scopo del test, la seconda parte dell'intervista prevedeva che l'informatore venisse sollecitato a conversare a proposito dello statuto linguistico delle denominazioni dei Comuni valdostani: l'obiettivo era in questo caso quello di far affiorare, nell'interazione con l'intervistatore e a partire da alcune domande-stimolo, le opinioni metalinguistiche e meta-sociolinguistiche a proposito di cause ed effetti 
Toponimi e identità: la percezione dei parlanti valdostani (Prima Parte)

L. ReVELLI della variazione; di attivare valutazioni a proposito della sovrabbondanza di pronunce e ipotesi relative alle rappresentazioni di correttezza; di sollecitare consapevolezza rispetto alla coerenza dei comportamenti propri e altrui; di rendere esplicita l'adesione a norme, canoni, paradigmi di riferimento; di far emergere eventuali pregiudizi, stereotipi, cliché nella formulazione di giudizi di valore o disvalore.

Relativamente ai risultati della parte dell'inchiesta basata sull'elicitazione delle pronunce ci limitiamo qui a riferire che i tratti di oscillazione più rilevanti e diffusi hanno riguardato le alternanze accentuali, ossitone o parossitone, soprattutto nella pronuncia delle denominazioni bisillabe; il discontinuo trattamento di - e, - d, - $\mathrm{se}-\mathrm{z}$ finali, alternativamente pronunciate o omesse; vari adattamenti introdotti per la resa di suoni estranei al sistema fonologico dell'italiano invece implicati nel modello francese adottato per la grafia (ad esempio /y/ $>/ \mathrm{u} / ; / 3 />/ \mathrm{b} /$ ), le fluttuazioni nell'articolazione delle vocali seguite in una stessa sillaba da -m o -n, nasalizzate secondo il modello francese o più spesso pronunciate con una sequenza di vocale orale+consonante nasale, secondo la tipica pronuncia italiana del francese (Maturi 2006: 145). 


\section{Tabella 1}

\section{ESEMPI DI VARIAZIONE NELLA PRONUNCIA DEI TOPONIMI COMUNALI DELLA VALLE D'AOSTA}

Il riferimento al modello di pronuncia francoprovenzale è indicato tra parentesi quadre, con la riproduzione dell'indicazione fornita dal Nouveau dictionnaire de patois valdôtain di Aimé Chenal e Raymond Vautherin (Quart, Musumeci, $1997=$ NDPV)

\begin{tabular}{|c|c|}
\hline $\begin{array}{l}\text { posizionamento } \\
\text { dell'accento tonico } \\
\text { in toponimi bisillabi }\end{array}$ & $\begin{array}{l}\text { FÉNIS [NDPV: "fe-nìs ou fen-ìs, n nasal']: /'fenis/: } 62 \% \text {; /fe'nis/: } 38 \% \text {. Nessuna } \\
\text { correlazione di rilievo emerge in rapporto alle lingue utilizzate in ambito familiare, né } \\
\text { in relazione ai luoghi di nascita e residenza o al titolo di studio. A prescindere dalla } \\
\text { scelte d'accento, la -n- non è mai nasalizzata e la - s finale è sempre pronunciata, } \\
\text { come sorda. } \\
\text { DONNAS [NDPV: "do-nàs"]: /'donnats/ ( } 15 \text { occ.), /'donnas/ ( } 49 \text { occ.), /'dənna/ ( } 2 \\
\text { occ.), /don'nas/ ( } 64 \text { occ.), /don'na/ ( } 1 \text { occ.) Accentazione parossitona: } 49 \% \text {; ossito- } \\
\text { na: } 51 \% \text {.Tendono a privilegiare le varianti ossitone i giovani più degli anziani, i quali } \\
\text { conservano in alcuni casi la pronuncia terminante con -ts, probabile residuo della gra- } \\
\text { fia Donnaz in uso fino al } 1976 \text {. } \\
\text { VERRÈS [NDPV: s.v. Verès: "ve-rès"]: /'verres/: } 75 \text { occ. La pronuncia parossitona è } \\
\text { preferita anche fra coloro che dichiarano di utilizzare in famiglia il patois (18 occ.; ossi- } \\
\text { tona: } 15 \text { occ.). }\end{array}$ \\
\hline $\begin{array}{l}\text { trattamento } \\
\text { del digramma qu }\end{array}$ & $\begin{array}{l}\text { QUART [NDPV: s.v. Quar "kàr"]: (/kwart/: } 56 \% \text {; / kar } /: 44 \% \text { ). } \\
\text { A privilegiare la pronuncia italianizzante sono prevalentemente i parlanti dialettofoni, } \\
\text { specie se collocati nella fascia d'età oltre i cinquant'anni. I modelli televisivi escludono } \\
\text { invece la pronuncia della semivocale e della consonante finale, che anche alcuni giova- } \\
\text { ni esplicitamente stigmatizzano, facendo riferimento alla norma francese veicolata } \\
\text { dalla scuola. }\end{array}$ \\
\hline trattamento $d i-d$ & $\begin{array}{l}\text { BARD [NDPV: s.v. Bar: "bàr"]: /bard/: } 20 \text { occ. }(17 \%) \text {; /bar/: } 83 \% \text {. L'esito /bard/, } \\
\text { che non è raro sentire anche nei modelli televisivi locali, è stato selezionato in tre casi } \\
\text { da parlanti dialettofoni, in quattro da parlanti provenienti da altre regioni. Alcuni testi- } \\
\text { moni condotti a riflettere sulla loro scelta hanno affermato di usare alternativamente le } \\
\text { due forme antagoniste a seconda della collocazione del toponimo all'interno della } \\
\text { frase, e quindi sulla spinta di ragioni eufoniche o fonosintattiche }\end{array}$ \\
\hline trattamento di-z & $\begin{array}{l}\text { BIONAZ [NDPV: s.v. Biòna: "bi-ò-na"]: /'bjona/: } 73 \% \text {; /'bjonats/: } 35 \text { occ. Soltanto } \\
\text { otto parlanti che hanno optato per la variante terminante in -ts applicano poi lo stes- } \\
\text { so principio al tipo PERLOZ [NDPV: s.v. Perlo: "pèr-lo"]: //perlo/: } 75 \text { occ.; /per- } \\
\text { lots/: } 32 \text { occ.; /perlo/: } 4 \text { occ. Tendenzialmente sembra di osservare una predilezione } \\
\text { della pronuncia con -ts nelle persone più mature (età media: } 44 \text { anni; esiti vocalici: } 36 \\
\text { anni), mentre non si riscontrano correlazioni evidenti con le abitudini linguistiche del- } \\
\text { l'ambito domestico né con il paese di nascita o l'area di provenienza }\end{array}$ \\
\hline trattamento di-e & 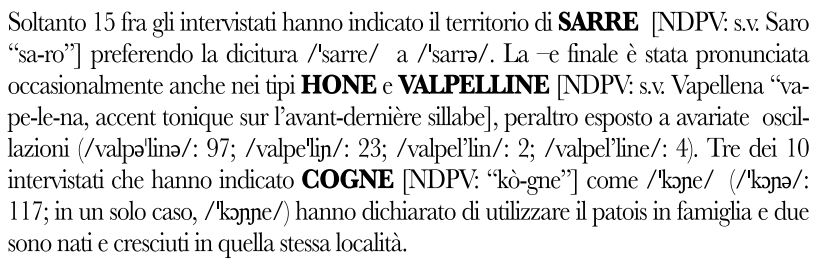 \\
\hline
\end{tabular}


Toponimi e identità: la percezione dei parlanti valdostani (Prima Parte) L. ReVelui

\section{LA VARIAZIONE SECONDO I PARLANTI}

Rimandando a Revelli 2013 per un più analitico resoconto dei fenomeni di variazione registrati e delle correlazioni esistenti tra le scelte di pronuncia e alcune variabili sociolinguistiche rivelatesi significative, riportiamo nella Tabella 1 qualche esempio utile a contestualizzare la dimensione all'interno della quale si collocano i fenomeni a proposito dei quali i parlanti consultati hanno espresso le opinioni che nelle prossime pagine ci proponiamo di commentare, avvertendo che:

- gli intervistati vengono indicati, tra parentesi quadre, attraverso la sigla P seguita da un codice numerico convenzionale;

- nella riproduzione delle battute di parlato i toponimi sono rappresentati tra barre oblique, in alfabeto IPA, con introduzione del segno $/ /$ prima della sillaba accentata;

- pause, reticenze ed esitazioni sono riprodotte attraverso i tre punti / .../, a prescindere dalla durata;

- riempitivi e vocalizzi non verbali sono resi in modo approssimato, attraverso soluzioni grafiche come mhm, eh, boh;

- le grafie ufficiali per i 73 Comuni della Valle d'Aosta che si aggiungono a quello di Aosta-Aoste, unico a possedere doppia denominazione ufficiale, sono, in ordine alfabetico, le seguenti (6):

Allein, Antey-Saint-André, Arnad, Arvier, Avise, Ayas, Aymavilles, Bard, Bionaz, Brissogne, Brusson, Challand-Saint-Anselme, Challand-Saint-Victor, Chambave, Chamois, Champdepraz, Champorcher, Charvensod, Châtillon, Cogne, Courmayeur, Donnas, Doues, Emarèse, Etroubles, Fénis, Fontainemore, Gaby, Gignod, Gressan, Gressoney-La-Trinité, Gressoney-Saint-Jean, Hône, Introd, Issime, Issogne, Fovençan, La Magdeleine, La Salle, La Thuile, Lillianes, Montiovet, Morgex, Nus, Ollomont, Oyace, Perloz, Pollein, Pontboset, Pontey, PontSaint-Martin, Pré-Saint-Didier, Quart, Rhêmes-Notre-Dame, Rhêmes-SaintGeorges, Roisan, Saint-Christophe, Saint-Denis, Saint-Marcel, Saint-Nicolas, Saint-Oyen, Saint-Pierre, Saint-Rhémy-en-Bosses, Saint-Vincent, Sarre, Torgnon, Valgrisenche, Valpelline, Valsavarenche, Valtournenche, Verrayes, Verrès, Villenewve.

Una prima seppur provvisoria ripartizione interna del campione degli intervistati può essere operata sulla base delle risposte alla domanda: "Secondo lei il fatto che i nomi dei Comuni vengano pronunciati in modi diversi è un fatto positivo, negativo o che non ha importanza?".

Il $42 \%$ del campione dichiara di attribuire al fenomeno scarso rilievo ([P32]: non ha nessuna importanza: basta farsi capire), considerandolo come radicato nell'area ([P27] mi accorgevo anche quando andavo a fare l'umiversità che già in treno quando non c'erano ancora quelle cose come adesso... le voci registrate... che quindi teoricamente lo dicono guisto maaa... a seconda di chi c'era una volta diceva /verres/ una volta diceva /ver'res/, /sanven'san/ o /senven'san/), o registrandone l'esistenza con rassegnazione o indulgenza: 
Toponimi e identità: la percezione dei parlanti valdostani (Prima Parte)

L. ReVELLI
[P118]: secondo me... sì, boh, c'è un po' una confusione ... non so, forse un po' di ignoranza, però a me... non mi sembra cosi grave... cioè: mi sembrerebbe grave se da qualche parte ci fosse... un riferimento che tutti sanno... che si chiama /'verres/ piuttosto che /ver'res/, però invece siccome mi sembra che a livello generale ci sia una tale confusione, allora dici: va beh.

[P33]: magari per quelli del posto cambia qualcosa perché sanno come andrebbe pronunciato quindi dà fastidio se qualcuno lo pronuncia in modo diverso, però cosi ... non credo che sia una cosa ... rilevante.

[P132]: per quanto riguarda /kwart/, probabilmente bisognerebbe dire / $\mathrm{kar} /$, perché ... perché è in francese. Poi sono io il primo che dico /kwart/. Nel caso di /'fenis/... sinceramente non so se è guisto /'fenis/ o /fe'nis/, però proprio appunto perché non do importanza. Non è che se uno.... l'mportante è che ci si capisca.

Atteggiamenti di malcelata o esplicita ostilità nei confronti dell'oscillazione delle pronunce sono invece individuabili in un terzo delle risposte $(34 \%)$, e si manifestano prevalentemente attraverso commenti di negazione della possibilità che esistano varianti dotate di pari dignità:

[P2]: non è che vengano pronunciati in maniera diversa: vengono pronunciati in modo giusto o sbagliato.

[P11]: se un posto ha un nome, se s'ha deggl accenti bisogna rispettarli. Ci sono degh accenti nelle parole, non è che uno se li imventa!

[P 77]: se cè una pronuncia dev'essere quella.

[P119]: io sono uno molto preciso e secondo me è negativo che ci sia questa promiscuità di pronunce, perché comunque... se mhm... se un Comune ha quel nome, dev'essere pronunciato in una certa maniera.

I giudizi più categorici - non necessariamente provenienti da parlanti endogeni - fanno riferimento a due principi fondamentali: la sacralità dello statuto del nome proprio, sentito come inviolabile e intraducibile ([P134] è importante, perché tipo... se mi chiamano Dàniela invece che Danièla... beh: la pronuncia è importante) e il riferimento a norme grammaticali, evocate però in modo astratto e indeterminato, senza che sia chiaro quale sia il codice del repertorio e le specifiche convenzioni cui viene fatto riferimento. Così, ad esempio, [P6] afferma: I nomi dovrebbero essere giusti, cioè....̀̀ grammatica.

Alcuni motivano la loro disapprovazione per la compresenza di soluzioni alternative esprimendo fastidio per l'intersecarsi dei diversi codici disponibili ([P6] secondo me questa varietà delle pronunce è un casino. Per esempio mia madre sbaglia perché pronuncia in italiano come in dialetto) o lamentando inconvenienti comunicativi conseguenti a una mancata mutua comprensibilità ([P 88] la pronuncia dei nomi dei paesi cambia tanto. La gente non si capisce. Non mi piace.) che i più danno invece per scontata ([P38]: /kwart/ o /kar/: ci capiamo lo stesso ).

Il $24 \%$ degli intervistati guarda con favore alla varietà e all'assenza di convenzioni condivise ([P127]: mi diverte lidea che nessuno sappia esattamen- 
Toponimi e identità: la percezione dei parlanti valdostani (Prima Parte) L. REVELLI

\section{LEALTÀ \\ LINGUISTICA E APPARTENENZA CULTURALE}

te come pronunciare ... diciamo che mi diverte eh lidea di non sapere dove sta la verità, se sbaglio io o sbaglia lui o sbaglia l'altro, quindi... va bene cosi).

L'eterogeneità delle pronunce è interpretata come veicolo di una libertà che consente a ciascuno di selezionare le varianti più consone al proprio idioletto ([P 76] Secondo me la varietà è bella: con la pronuncia ... collochi la persona al suo posto): in questo senso, alcuni - manifestando un senso della diatopia interna (Telmon 2002) che, come vedremo oltre, si è rivelato in generale molto spiccato e diffuso - fanno riferimento alla storica frammentazione dialettale del territorio: [P133] [la varietà di pronuncia] è bella perché è molto legata al linguaggio del territorio, che a seconda del patois che solitamente viene utilizzato - perché son tutti diwersi- allora la pronuncia cambia.

Non sempre le dichiarazioni di principio hanno trovato corrispondenza nelle successive fasi della conversazione: anche da parte dei parlanti maggiormente aperti alla variazione sono, infatti, spesso emersi atteggiamenti rivelatori di sentimenti di imbarazzo nei confronti di una francofonia o dialettofonia asserita ma vissuta con conflittualità rispetto agli usi spontanei:

[P98]: a me mi è sempre venuto da dire /fenis/invece che /feni/, / donnas/ invece che /don'na/, e invece... invece bisognerebbe dargli l'accento sull'ultima sillaba sennò ... ehm... beh, perché io ci tengo ad essere valdostana e mi piace che... che vengano pronunciati nel modo giusto, ecco.

[P129]: se io dico /kar/ è perché mi sforzo per non dire / kwart /.

Lo iato tra intenzione ed esecuzione, tra lealtà al territorio e spontaneità della produzione linguistica provoca in non pochi degli intervistati un senso di inadeguatezza che si concretizza nell'uso frequente di enunciazioni ottative e espressioni formulate attraverso verbi di tempo condizionale:

[P6]: noi dovremmo essere una regione francoprovenzale e quindi dovremmo essere più ... forti ... cioè: ci doorrebbe venire ... cioè ... eh, più spontaneo dire / $\mathrm{kar} /$, /fe'nis/... E invece non ci riusciamo.

[P20]: (il nome di un paese) uno deve dirlo come ... secondo me... com'è il più giusto. Secondo me ogni popolazione del paese dovrebbe sapere il modo giusto di dirlo.

[P56]: sono nomi francesi e quindi dovrebbero avere una pronuncia francese e invece in realtà qui ad Aosta spesso sbagliamo. Anchio personalmente sbaglio.

[P67]: adesso ... cioè io ... da parte mia preferisco la pronuncia valdostana, francese, eh... così...almeno... secondo me. Poi ciascuno... Per me è un po' negativo [che ci siano pronunce diverse] perché comunque... ciò̀... siamo anche ... francofoni, quindi dovremmo...

[P84]: sarebbe giusto saperlo dire ....come si dice... cioè... siamo un paese che confina con la Francia quindi i paesini bisognerebbe... almeno... penso... secondo me, eh... bisognerebbe saperli dire nel modo giusto. 
Toponimi e identità: la percezione dei parlanti valdostani (Prima Parte) L. ReVELLI

\section{LA NORMA (IN)DESIDERATA}

[P 101]: è che siamo forse un po' ignoranti su alcune cose... nel senso che ... mhm, è un modo di dire, però in realtà non conosciamo forse ... abbastanza bene... i nomi dei posti per pronunciarli nel modo giusto, non so come dire ... eh, è un po' un discorso di... cioè ... perché son nomi francesi per cui dovresti dargli l'intonazione, e invece col tempo... tipo Mon' wiove: l'hanno italianizzato.

In questo contesto, ma non necessariamente stigmatizzando il fenomeno, diversi parlanti riconoscono di non adottare, per lo meno non sempre, comportamenti omogenei e di non scegliere necessariamente le soluzioni che pure ritengono più "corrette":

[P10]: / $\mathrm{kar} /$ si deve dire, e invece noi diciamo tutti / kwart/.

[P35]: io stessa una volta dico in un modo e la volta dopo dico in un altro. Non so come si dica nella realtà.

[P50]: vedo che ... alcune volte dico $/$ fenis/, altre volte /fenis/, ma ... non so perché: me ne accorgo che cambio.

[P55]: io li dico a seconda di come ... bhò... non lo so, dipende... anche dipende da con chi sto parlando... dipende anche dal contesto, dalla frase.

[P66]: si, eh... succede che tipo /monzove/ diventa /mon'bove/, /'donnas/... /don'nas/ : diciamo che andrebbero pronunciati bene alla francese, però... vengono un po' italianizzati. Diciamo che mi piacerebbe pronunciarli sempre giusti, però... faccio un po' come capita.

[P 97]: dico più che altro /verres/, ma dipende ... eh... dipende dai giorni.

[P 113]: son già io la prima che pronuncio male! /'fenis/: io non dico mai /fe'nis/, dico sempre /ffenis/! O /kar/, io lo chiamo sempre /kwart/.

L'assenza di regolarità nelle pronunce viene da molti ricondotta alla mancanza di canoni fonologici e modelli normativi di sicuro riferimento. Anche chi si dichiara maggiormente contrariato dalle oscillazioni manifesta poi dubbi e incertezze nell'orientarsi fra le diverse soluzioni disponibili:

[P14]: una cosa che noto è che molti dicono / $\mathrm{kwart} /$ invece che ... ciò̀ ... non so ... ma penso che sia giusto dire $/ \mathrm{kar} /$.

[P29]: mi dà fastidio non sapere se è giusto /'verres/ o /ver'res/, per esempio.

[P30]: io ... da quello che so... è che comunque ad esempio /'biona/ ... vedi, io dico /'Biona/, ma in teoria si dovrebbe dire /'bionaz/. O no?'

[P37]: è che non si sa la pronuncia giusta, cioè ... la si diceee così... alle volte sbaglio anchio: /'donnas/ o /don'nas/?

[P53]: io non so mai come dirli, dove mettere l'accento, se dire la esse alla fine.

[P 85]: per esempio io dico/'fenis/ anche se so che sarebbe /fe'ni/. No, non so in realtà. Io dico /'fenis/. /fe'ni/ non mi sembrerebbe giusto. Comunque ci sono dei nomi di certi Comuni che vengono detti in una maniera... tipo /'donnas/ che molti dicono /'donna/ o /don'nas/ .

[P 93]: a me mi viene da dirli alla francese... perché mi sembra più giusto... però poi non so se è giusto. 
Toponimi e identità: la percezione dei parlanti valdostani (Prima Parte)

L. ReVELLI
[P115]: a volte è un po' complicato, tipo / kar/e / kwart/: alcuni dicono... ma anche chi ci abita... alcuni dicono in un modo, altri in un altro.... A me...ciò̀... io tutte le volte mi pongo il quesito, e dico: ma come lo devo dire? $O$ se no... non so... tipo /'perlo/: alcuni dicono /'perloz/, chi ci abita anche. A me piacerebbe sapere di preciso come va detto.

[P120]: delle volte mi chiedo quale sia la pronuncia giusta, perché sicuramente ce n’è una giusta.

Lincertezza attiva, soprattutto ma non soltanto in chi proviene da fuori regione, dispositivi di orientamento sugli interlocutori e sulle loro aspettative:

[P 111]: da persona che è qua da 21 anni ... ho dovuto cercare di capire - un po' come con i cognomi, del resto - ho dovuto cercare di capire cosa si aspettava la maggior parte della gente nella pronuncia dei Comuni $[. .$.$] Per i$ Comuni in realtà io cerco di rispettare da un lato quella che è la pronuncia giusta, visto che in molti casi son nomi francesi, e dall'altro capire un po' come vogliono che vengano pronunciati qua, per cui non so... io dico / kar/, però molta gente dice / kwart/.

[P 101]: io dico a volte /'donnaz/, a volte /don'nas/, perché ... perché so che la gente di li vuole che si dica/'donnaz/ in quanto... in realtà è cosi che loro lo pronunciano, per cui... eh, dipende un po'.

[P127]: a volte io stessa prima di pronunciare il nome di un Comune mhm mentalmente eh... ci penso, e poi dico: ma l'aurò detto giusto? Perché in effetti lo sento magari pronunciare in modo diverso da altri.

Il senso di smarrimento provocato dalla sovrabbondanza delle varianti induce alcuni a desiderare regolarità e modelli univoci, o addirittura a formulare l'auspicio che si possa pervenire a una codificazione imposta centralmente:

[P18]: a me piacerebbe che $i$ paesi venissero chiamati col loro nome e che anch'io potessi sapere esattamente qual è questo nome: che ci fosse .... eh ... una pronuncia prestabilita.

[P28; ]: la varietà crea confusione. Anche dal punto di vista istituzionale. Proprio ieri sera parlavamo che /ar'na/ era /ar'naz/ fino aaaa... all'Ottanta forse, e poi è diventato /arnad/. Cioè: sarebbe il caso di definire una volta per tutte... E /'verres/, anche, che /ver'ès/ lo chiama pochissima gente.

[P38]: sarebbe bello che ci fosse una pronuncia unica per tutti.

[P40]: io trovo piuttosto negativo che un paese proprio vicino... che abbiano una pronuncia ... che uno lo dice in un modo uno in un altro ... non si capisce.

[P81]: magari anche per chi non parla patois o francese sarebbe più comprensibile cioè più chiaro avere un'unica pronuncia.

[P131]: direi che dovrebbe essere data una linea d'indinizzo sulla lettura corretta, però probabilmente $i$ locali lo pronunciano nel modo giusto mentre le persone non abitanti nel ... nel paese... non conoscono esattamente la pronuncia, se c'è l'accento sulla e piuttosto che se si pronuncia la zeta finale o meno. Però se ci fosse un ordine sarebbe sicuramente produttivo per $i$ turisti ma nel contempo anche per gli abitanti per- 
Toponimi e identità: la percezione dei parlanti valdostani (Prima Parte) L. ReVELLI

\section{L'IDIOSINCRASIA} PER LE PRONUNCE 'SBAGLIATE' ché vengono riconosciute le loro... caratteristiche ... anche fonetiche.

Il denominatore che accomuna i parlanti che si auspicherebbero una ristandardizzazione delle pronunce lascia però trapelare presupposti diversi: se i più danno per implicito o scontato che un modello prescrittivo debba fare riferimento al francese ([P 75] sarebbe bello che fossero pronunciati tutti in francese uguale), alcuni fanno invece riferimento ai canoni del francoprovenzale ([P 102] bisognerebbe pronunciarli alla francoprovenzale; $[\mathrm{P} 110]$ Io credo che la cosa più giusta sarebbe fare riferimento al nome in patois, $e$ poi eventualmente riproporlo in termini... francesizzandolo, per dargli una grafia), mentre le voci inclini a un adattamento verso l'italiano risultano decisamente fuori dal coro ([P 88] Poi anche siamo italiani: i nomi doorebbero essere italiani, non francesi. Noi non c'entriamo niente con la Francia).

Rarissimi sono per converso gli atteggiamenti di avversione nei confronti dei tentativi di standardizzazione, sentiti come inutili forzature:

[P21]: io odio le omologazioni: /ar'na/ è giusto che ognuno lo pronunci, eh... . Come vuole]. Adesso rifanno le targhe ad Aosta per avere il toponimo preciso e perfetto con la targhetta e invece è bello andare a scoprire come... in qualche modo un tempo si metteva la zeta, un tempo si metteva la di: è la cosa più bella! Adesso no: tutto standardizzato.

Non tutti gli scarti rispetto alle pronunce considerate desiderabili sono giudicati con pari severità. In linea di massima, i commenti negativi si concentrano su determinati tratti percepiti come marcati perché corrispondenti a pronunce che si distanziano dai modelli fonologici del francese e del francoprovenzale, ossia ritenuti compromessi dall'adattamento di suoni, come le vocali nasali e quelle turbate, verso rese semplificate che trovano corrispondenza nell'inventario fonematico dell'italiano: [P24]: quando senti dire al telegiornale /curma ier/ ti da fastidio, perché noi siamo in Valle d'Aosta, almeno la nostra tradizione, le nostre... i nostri valori... cioè... i nomi francesi che abbiamo nella nostra Valle dobbiamo tenerli francesi.

[P 110]: quello che mi disturba è quando c'è un evidente errore, tipo chiamare /sanven'san/ invece di /senven'san/: quello mi disturba moltissimo.

[P73]: io non ci faccio caso, nel senso che sono abituata a sentire italiano e patois continuamente, per cui non ci faccio caso sinceramente se cambia la pronuncia, cioè lo sento se so che è sbagliata. Nel senso, tipo /sanven'san/ so che è sbaghiata ma la pronunciano quelli che non sanno il francese tipo, che ne so, calabresi immigrati che non l'hanno mai studiato. Sennò non ci faccio caso: solo se è sbagliata me ne accorgo. Se sono abituata a sentirla in patois... non ci faccio caso; in italiano neanche; in fran... - cioè, eh, in italiano: i valdostani che parlano in francese - mi accorgo che mi dà fastidio se la pronunciano sbagliata quelli che non sanno il francese.

[P120]: ad esempio quando dicono /nus/invece che /nys/ mi dà terribilmente fastidio.

L'intolleranza è maggiore quando ad adottare una pronuncia disalli- 
Toponimi e identità: la percezione dei parlanti valdostani (Prima Parte)

L. ReVELLI neata sono persone residenti in Valle d'Aosta ([P23]: Un conto è il milanese che lo pronuncia sbagliato, un conto è il valdostano che dice /mon'gove/ per /montzo've/), e soprattutto se si tratta di personalità che rivestono ruoli istituzionali: [P34]: quando sento il sindaco di /en'tro/ che dice /in'tro/ o quello

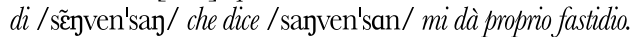

Oggetto di diffusa disapprovazione sono, inoltre, i giornalisti delle testate televisive locali:

[P49]: secondo me la cosa più critica è che per esempio al TG-Regione i nomi der comuni vengano pronunciati in mamiera scorretta che ... voglio dire... dovrebbe essere un esempio eh ... e poi ci stupiamo che la gente comune non sappia pronunciarli, ma è ovrio!

[P 106]: francamente non mi sono mai posto il problema di come io pronuncio, però devo dire che mi dà molto fastidio se per esempio sento un giornalista del TG3 che massacra il nome di un paese.

Una minoranza degli intervistati dichiara di sanzionare, attraverso esplicite correzioni, gli interlocutori che impiegano varianti di pronuncia ritenute improprie:

[P5]: a volte con gli amici litighiamo per come è più giusto dire.

[P24]: però tipo io ho una compagna di /'fenis/ che si arrabbia quando noi diciamo /'Fenis/, perché secondo lei si dice /fe'nis/.

[P49]: io tendo a correggere la gente che dice ... degli errori.

[P52]: per me la pronuncia non ha importanza, ma per mia mamma ne ha un sacco: lei mi dice sempre di dire /fe'nis/, /kar/ ...

[P 72]: se è gente che conosco li correggo, cioè ... che ne so... se è gente di famiglia li correggo. Chiaro che se è gente ... estranea .... allora no.

[P 91]: io probabilmente prima li pronunciavo malamente, ad esempio /fénis/ lo pronunciawo /'fenis/. Poi mi è stato fatto presente da un professore e da allora sono molto più attenta, anche se gli stessi abitanti di /'fenis/ quando parlano in italiano sbagliano la pronuncia perché dicono /'fenis/ anziché /fe'nis/ ... Però non è una cosa sentita in paese, nessuno ti correggerebbe mai

[P 92]: moltifanno cosi... eh, dicono /sar/ o /sarre/. Per me, non essendo nata qua, io come lo dico lo dico, non m'importa tanto, però... vedo che c'è gente qua, del posto, valdostani che a volte ti correggono... ho trovato alcune persone che mi hanno corretto, e però non si sa mai come si deve dire. Ad esempio: /fenis/ o /fe'nis/?

[P 96]: io correggo quando sento dire sbagliato, anche i miei figli li correggo, perché... non mi piace sentir dire tipo /verres/: io ci tengo, perché poi alla fine ... altrimenti è italianizzato.

[P116]: i valdostani doc, ad esempio ... cioè... io queste discussioni ce l'ho sempre a casa perché mio papà lui dice: "no, assolutamente non dire /ponte/: è /pont/ [Pont-Saint-Martin), e si deve pronunciare correttamente" però no... io trovo che invece sia mhm ... normale.

Soltanto due degli intervistati sottolineano la distinzione tra oralità e scrittura, attribuendo agli scarti ortografici un peso decisamente maggiore: 
Toponimi e identità: la percezione dei parlanti valdostani (Prima Parte)

L. ReVELLI

[P42]: la pronuncia dipende dalle zone, dai dialetti, dalla lingua ... comunque il nome è sempre quello, finché si scrive sempre uguale, ognuno ... è sempre la stessa cosa. A me non disturba. Se proprio non cambiano la scrittura, è quella la cosa importante.

[P 110]: mi disturba molto di più quando la grafia è sbagliata.

Che la reattività dei parlanti sia molto minore di fronte alle realizzazioni orali rispetto a quelle scritte è tuttavia dimostrato dalla frequenza e dai toni con cui veri e propri collezionisti di "errori" denunciano, attraverso lettere ai giornali, pagine web dedicate (ad esempio: www.facebook.com/valdaosta.vda) o anche pubblicazioni locali la presenza di pecche ortografiche nelle scritture esposte (cartelli stradali, insegne, manifesti), pubbliche e istituzionali.

FINE PARTE PRIMA (à suivre!)

\section{NOTE}

(1) Legge Regionale n. 4 del 28 febbraio 2011: Modificazioni alla legge regionale 9 dicembre 1976, $n$. 61 (Denominazione ufficiale dei comuni della Valle d'Aosta e norme per la tutela della toponomastica locale).

(2) Annexe A à la délibération du Gouvernement régional n 028 du 20 avril 2012: «les graphies s'inspirent de la tradition valdôtaine. Il s'agit donc de graphies françaises tenant compte, plus particulièrement, des caractères propres à l'aire francoprovençale, même quand elles ne rentrent pas dans les standards du français ». Più in particolare si prevede che le grafie debbano « conserver le $\mathrm{z}$ final, non prononcé après les voyelles 0 - $a-o u$ finales atones [...]; conserver le $\mathrm{x}$ final non prononcé, quand il existe dans la tradition graphique, pour les voyelles finales toniques é et $i[\ldots]$; privilégier la consonne $d$ après les voyelles finales toniques $a$ o et $o u[\ldots]$; utiliser la séquence ey en position finale quand, en patois, la prononciation est $\ddot{e}[$ [...]; insérer les traits d'union quand le nom à officialiser est composé [...]; réinsérer l'article (souvent négligé dans les sources écrites) quand il est présent dans l'expression patoise»

(3) Giovani e giovani adulti (18-31 anni), adulti (32-50 anni), tardo-adulti e anziani (51-88 anni).

(4) Luoghi di nascita: Valle d'Aosta: 73\%; altra regione italiana: 23\%; Stato estero: 4\%; domicilio: Aosta: 29\%; altri Comuni: 71\%.

$35 \%$

(5) Laurea e postlauream: $34 \%$; secondaria superiore: $31 \%$; secondaria inferiore+elementare:

(6) Le popolazioni walser della valle del Lys sono autorizzate dalla Legge Regionale n. 61 del 9 dicembre a «ajouter aux dénominations officielles les dénominations, selon les variantes locales, titsch et töitschu». 